\title{
Ernährung, Bewegung und Gesundheit
}

\author{
Die Weltgesundheitsorganisation ist weltweit um die Prävention chronischer \\ Krankheiten bemüht. Dafür hat sie im Jahr 2004 eine Strategie verabschiedet, \\ die Ernährung und Bewegung insbesondere von Kindern und Jugendlichen in den \\ Mittelpunkt von Aktivitäten stellt.
}

$\mathrm{D}$ Von Ingrid Keller er Weltgesundheitsbericht der Weltgesundheitsorganisation (WHO) von 2002 ,Risiken senken - gesundes Leben fördern" hat verschiedene Risikofaktoren und deren Einfluss auf Krankheits- und Todesraten untersucht. Danach sind, global gesehen, wichtige Risikofaktoren mit Ernährung und Bewegung verbunden. Speziell in den industrialisierten Ländern sind folgende Risikofaktoren für die meisten vorzeitigen Todes- und Krankheitsfälle verantwortlich: Bluthochdruck, Tabakkonsum, erhöhter Serumcholesterinspiegel, Übergewicht, zu geringer Obst- und Gemüseverzehr, Bewegungsarmut und Alkoholkonsum. Allein sechs dieser sieben Risikofaktoren (Alkohol eingeschlossen) sind abhängig von Ernährung und Bewegung (1). Die ökonomischen und sozialen Folgen der Krankheiten, welche durch diese Risikofaktoren hervorgerufen werden, sind immens und daher besteht dringender Handlungsbedarf auf allen Ebenen und Sektoren.

Eine gesunde Ernährung, Bewegung, und Verzicht aufs Rauchen können relativ schnell individuelle Risiken bei der Entstehung unterschiedlichster Krankheiten mindern und einen wichtigen Beitrag zum Erhalt von Gesundheit leisten. Veränderungen im Alltagsleben können hier helfen und auch Interventionen mit relativ moderaten Kosten zur Veränderung der Bevölkerungsverteilung von Risikofaktoren wie Bluthochdruck haben substanzielle Auswirkungen auf die Allgemeingesundheit.

\section{Globale Strategie}

Die Weltgesundheitsversammlung der Mitgliedstaaten der WHO hat im Jahr 2000 die globale Strategie zur Prävention von nicht übertragbaren Krankheiten verabschiedet. Chronische Krankheiten sind eine Priorität der WHO mit dem Schwerpunkt auf Prävention der drei wichtigsten Risikofaktoren: Ernährung, Bewegung und Ta- bakkonsum. Die Rahmenkonvention zur Tabakkontrolle tritt Ende Februar 2005 in Kraft (2). Der WHO-Bericht zum Thema Ernährung und Prävention von chronischen Krankheiten enthält überarbeitete Ernährungsrichtlinien (3).

Im Auftrag der Mitgliedstaaten hat die WHO eine globale Strategie zum Thema Ernährung und Bewegung erstellt. Diese Strategie wurde in Konsultation mit den Mitgliedsländern, Nicht-Regierungsorganisationen, globalen Vertretern der Lebensmittelindustrie, anderen Organisationen der Vereinten Nationen und Experten erarbeitet und ist im Mai 2004 von der Weltgesundheits-Versammlung verabschiedet worden (4). Diese Strategie basiert auf zwei Grundlagen. Die Prävention von nicht übertragbaren Krankheiten (NüK) muss die Risikofaktoren ansprechen, die mehrere NüK gleichzeitig beeinflussen und nicht auf die Prävention von einzelnen Krankheiten abzielen. Alle Sektoren und Akteure in den Bereichen Gesundheit, Ernährung, Bildung, Sport und Bewegung, Stadtplanung, Transport, Wirtschaft und Handel müssen einbezogen werden und beitragen. Nur so kann die Auswirkung und die Nachhaltigkeit von gesundheitsfördernden und präventiven Aktionen gewährleistet werden. Die Strategie beinhaltet Vorschläge für die Erarbeitung von Strategien, Richtlinien und konkreten Aktionen für Regierungen, internationale Organisationen, die Zivilgesellschaft und den privaten Sektor. Folgende Empfehlungen werden gemacht:

1. die Erarbeitung von nationalen Strategien und Richtlinien für Ernährung und Bewegung, welche auch eine bessere Bildung und öffentliches Bewusstsein über NüK und deren Risikofaktoren beinhalten sollten,

2. die Förderung einer gesunden Ernährung und Sport in der Schule, da in fast allen Ländern die Mehrheit der Kinder so erreicht werden kann, sowie Gesundheits- und Ernährungsunterricht,
3. multi-sektorale Politiken, um Bewegung zu fördern,

4. die Gewährleistung einer ausgewogenen und genauen Information für Konsumenten, unter anderem durch Lebensmittelkennzeichnung und angepasste Werbung/Verkaufsförderung von Lebensmitteln, besonders im Bezug auf Kinder,

5. die Untersuchung von Landwirtschaftspolitiken auf ihre mögliche Auswirkungen über das Lebensmittelangebot auf die Gesundheit,

6. finanzpolitische Instrumente, wie zum Beispiel Steuern oder Subventionen, die eine gesunde Ernährung und körperliche Bewegung fördern,

7. eine ständige Evaluierung und Bewertung von gesundheitsfördernden Aktion, sowie

8. mehr Investitionen in Forschungs- und Public Health Einrichtungen.

Die WHO arbeit jetzt an der Implementierung der Strategie. Auf der einen Seite werden alle Länder, die diese Strategie in nationale Politiken eingliedern möchten, von den WHO Regionalbüros unterstïtzt. Auf der anderen Seite werden auf internationaler Ebene weitere Richtlinien und Aktionsvorschläge erarbeitet, die den Mitgliedsstaaten helfen sollen, die Strategie umzusetzen. Dieses sind unter anderem die Erarbeitung von globalen Richtlinien für Sport und Bewegung, eine multi-sektorale Diskussion über Lebensmittelwerbung und Marketing besonders im Bezug auf Kinder und die Erarbeitung von Richtlinien für das Lebensmittelangebot sowie die Bewegungsförderung in der Schule. Die globale Strategie der WHO hat multi-sektorale und multi-disziplinäre Aktivitäten wie zum Beispiel eine Plattform für Ernährung und Bewegung inspiriert.

\section{Anmerkungen}

(1) WHO: World Health Report, Reducing Risks, promoting healthy life. Genf 2002. www.who.int/whr/en

(2) WHO: Framework Convention for Tobacco Control. Genf 2003. www.who.int/tobacco/en/

(3) WHO: Expert Report on Diet, Nutrition and the Prevention of Chronic Diseases. Genf 2003.

www.who.int/hpr/gs.who.fao.report.shtml

4) WHO: Global Strategy on Diet, Physical Activity and Health. Genf 2004.

\section{Die Autorin}

Ingrid Keller ist Technical Officer am Chronic Disease and Health Promotion Department der World Health Organization (WHO).

Kontakt: WH0, 20 Avenue Appia, CH-1211 Genf 27. Tel. 0041-22-7913448, E-Mail: kelleri@who.int 
(c) 20I0 Authors; licensee IÖW and oekom verlag. This is an article distributed under the terms of the Creative Commons Attribution Non-Commercial No Derivates License (http://creativecommons.org/licenses/by-nc-nd/3.o/), which permits unrestricted use, distribution, and reproduction in any medium, provided the original work is properly cited. 